\title{
Heterogeneity of tremor mechanisms assessed by tremor-related cortical potential in mice
}

Young-Gyun Park', Jee Hyun Choi ${ }^{2}$, Chungki Lee², Sehyun Kim³ ${ }^{3}$ Youngsoo Kim', Ki-Young Chang ${ }^{1}$, Sun Ha Paek ${ }^{5}$ and Daesoo Kim ${ }^{1 *}$

\begin{abstract}
Background: Identifying a neural circuit mechanism that is differentially involved in tremor would aid in the diagnosis and cure of such cases. Here, we demonstrate that tremor-related cortical potential (TRCP) is differentially expressed in two different mouse models of tremor.

Results: Hybrid tremor analysis of harmaline-induced and genetic tremor in mice revealed that two authentic tremor frequencies for each type of tremor were conserved and showed an opposite dependence on CaV3.1 T-type

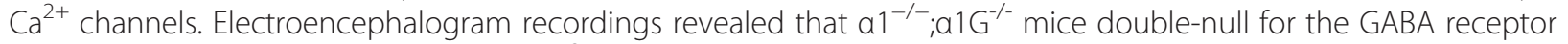
a1 subunit (Gabra1) and CaV3.1 T-type $\mathrm{Ca}^{2+}$ channels (Cacna1g), in which the tremor caused by the absence of Gabra1 is potentiated by the absence of Cacna1g, showed a coherent TRCP that exhibited an onset that preceded the initiation of behavioral tremor by 3 ms. However, harmaline-induced tremor, which is known to be abolished

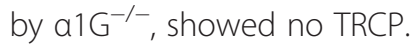

Conclusions: Our results demonstrate that the $\mathrm{a}^{-1-} ;{\mathrm{a} 1 \mathrm{G}^{-1-}}^{-}$double-knockout tremor model is useful for studying cortical mechanisms of tremor.

Keywords: Tremor mechanism, Cortical rhythm, Harmaline, T-type $\mathrm{Ca}^{2+}$ channels

\section{Background}

Tremor is an involuntary rhythmic oscillation of body parts $[1,2]$. Various mechanism ranges from reflex loop to central oscillation has been proposed for the origin of oscillatory activity generating tremor [1,2]. Animal models of tremor have been contributed to examine those hypotheses.

Harmaline is a plant-derived metabolite used to induce tremor in animals [3-5]. Harmaline tremor is an actioninduced tremor and coherent with pathological oscillation in olivocerebellar pathway, which propagates to reticulospinal pathway and entrains muscles with synchronized oscillation $[4,6,7]$. Either knockout of the Cacnalg gene encoding the $\mathrm{Ca}_{\mathrm{v}} 3.1$ channel $\alpha 1 \mathrm{G}$ subunit $\left(\alpha 1 \mathrm{G}^{-/-}\right.$mice) or pharmacological blockage of T-type $\mathrm{Ca}^{2+}$ channels in the inferior olive abolishes $10-15 \mathrm{~Hz}$ harmaline-induced tremor and the pathological oscillation [8], suggesting that

\footnotetext{
* Correspondence: daesoo@kaist.ac.kr

'Department of Biological Sciences, Korea Advanced Institute of Science and Technology (KAIST), 335 Gwahak-ro, Yuseong-gu, Daejeon 305-701, Republic of Korea

Full list of author information is available at the end of the article
}

activity of T-type calcium channel is critical for generating harmaline tremor.

T-type calcium channel has a contradictory role in the other model of action-induced tremor. In Gabra1 model of action tremor, knockout mice of $\alpha 1$ subunit of the $\gamma$ aminobutyric acid (GABA) receptor, additional knockout of $\alpha 1 \mathrm{G}$ enhances action-induced tremor [9]. Considering that T-type calcium channel is involved in thalamocortical oscillations $[10,11]$ and cortical lesion has no effect on harmaline tremor $[7,12]$, we hypothesized that two tremor models has different involvement of cortical mechanisms.

Here, using pharmacogenetic dissection of tremor frequencizes and high-resolution electroencephalograzphic (EEG) techniques [13] combining with event-related potential analysis(ERP) [14-17], we reveal that abnormal cortical rhythmicity in the form of tremor-related cortical potential (TRCP) is differentially expressed between harmaline and genetic models of action tremor. 


\section{Results}

Pharmacogenetic determination of tremor frequency independence

To test the independence of mechanism between harmaline and genetic tremors $\left(\alpha 1^{-/-}\right.$and $\left.\alpha 1^{-/-} ; \alpha 1 G^{-/-}\right)$[9], we performed pharmacogenetic experiment in which two types of tremor were produced in a mouse. We predicted two possibilities. The first is that the two tremor mechanisms interact to generate a hybrid tremor according to the dominant/recessive relationship between them. Alternatively, the two tremor frequencies may emerge independently, like the difference in behavior between two genes on separate chromosomes.

After harmaline administration ( $9 \mathrm{mg} / \mathrm{kg}$ ), wild-type (WT) mice exhibited only 10-15 Hz tremor (Figure 1A-C, left), whereas $\alpha 1^{-/-} ; \alpha 1 G^{-/-}$mice showed only $15-30 \mathrm{~Hz}$ tremor (Figure $1 \mathrm{~A}-\mathrm{C}$, right). In contrast, harmalinetreated $\alpha 1^{-1-}$ mice exhibited two separable band frequencies, 10-15 and 15-30 Hz, reflecting the coexistence of authentic harmaline and genetic tremor (Figure 1A-C, middle, and Figure 1D). Considering that the different tremor frequencies reflect the involvement of different neural circuit mechanisms [1,2], the two action tremor models appear to have distinct neural circuit mechanisms.

\section{Tremor-related cortical potentials in $a 1^{-/-} ;{\mathrm{a} 1 \mathrm{G}^{-/-}}^{-}$mice}

Using high-resolution EEG covering the whole frontal and occipital cortex (Figure 2A) and forelimb electromyography (EMG), we measured the cortical activity associated with forelimb tremor in $\alpha 1^{-/-} ; \alpha 1 G^{-/-}$mice. We isolated cortical potentials associated with tremor onset by averaging EEG activity corresponding to the first discharge of

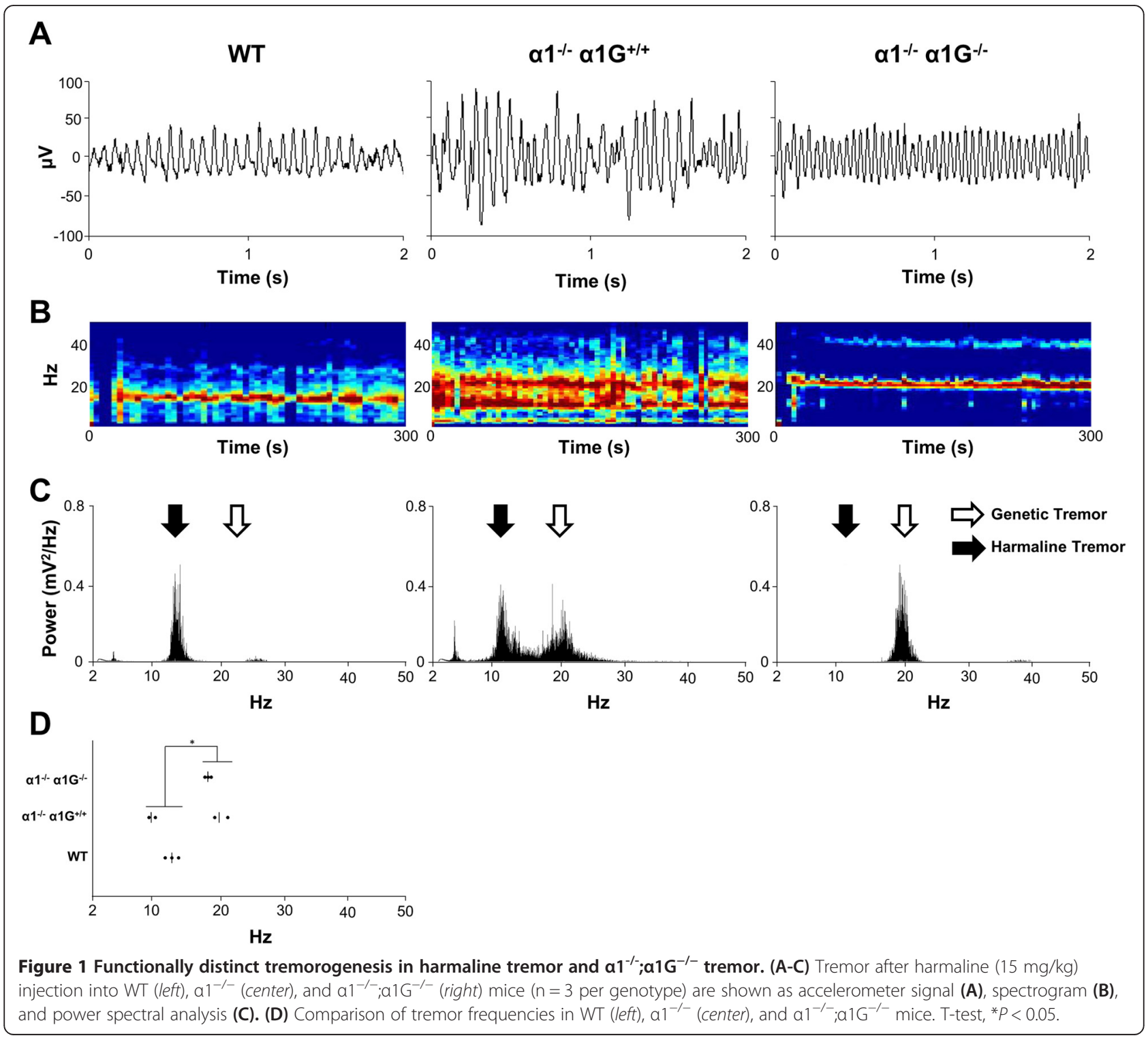




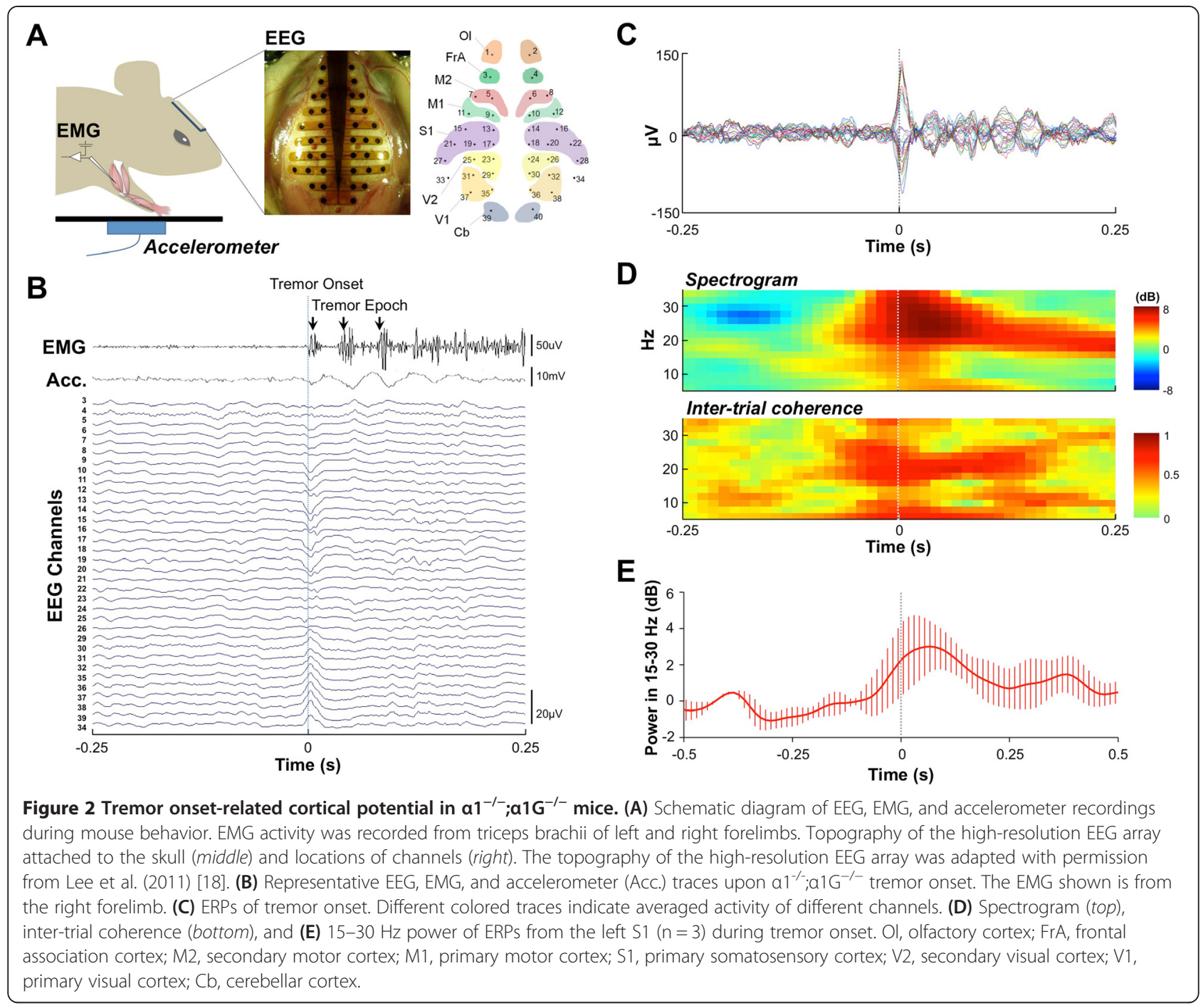

each tremor epoch. Tremor onset-related potentials in $\alpha 1^{-/-} ; \alpha 1 \mathrm{G}^{-/-}$mice initiated approximately $3 \mathrm{~ms}$ prior to behavioral tremor onset and were followed by $15-30 \mathrm{~Hz}$ oscillations corresponding to the accompanying muscular tremor discharges (Figure $2 \mathrm{~B}$ and $\mathrm{C}$ ). The resulting TRCPs were phase-locked to the $15-30 \mathrm{~Hz}$ tremor (Figure $2 \mathrm{~B}$ and $\mathrm{C}$ ), although their amplitudes and phases were variable among channels (Figure $2 \mathrm{~B}$ and $\mathrm{C}$ ), indicating the differential involvement of cortical subdomains. Power spectral analyses also confirmed that TRCPs were initiated upon tremor onset (Figure 2D, upper, and 2E) and coincided with tremor events (Figure 2D, lower). These results indicate that the $\alpha 1^{-/-} ; \alpha 1 G^{-/-}$tremor includes the cortical circuit mechanism, although the origin remains unknown.

\section{No TRCPs in harmaline tremor}

We then compared TRCPs in the two tremor models. When we aligned and averaged EEG traces along the timing of tremor discharges in $\alpha 1^{-1-} ; \alpha 1 \mathrm{G}^{-/-}$mice, TRCPs were obtained as an oscillatory pattern (Figure $3 \mathrm{~A}$, left). However, TRCPs were not found in harmaline tremor (Figure 3A, right) or in non-tremor contractions of $\alpha 1^{-/-} ; \alpha 1 \mathrm{G}^{-/-}$mice (Figure 3A, middle). The power and coherence of TRCPs were significantly lower in harmaline tremor than in $\alpha 1^{-/-} ; \alpha 1 G^{-/-}$tremor (Figure 3B-D). These results suggest that TRCPs can be a robust measure for discriminating these two types of tremor models.

\section{Discussion}

Essential tremor is a neurological condition characterized by action-induced tremors [2,19]. Considerable evidence suggests that essential tremor is heterogeneous with respect to affected body parts, tremor frequency, and drug sensitivity [20-24]. The heterogeneity appears to reflect the involvement of different neural substrates. While some studies have reported that abnormal cortical rhythms are associated with tremor in a subset of essential 


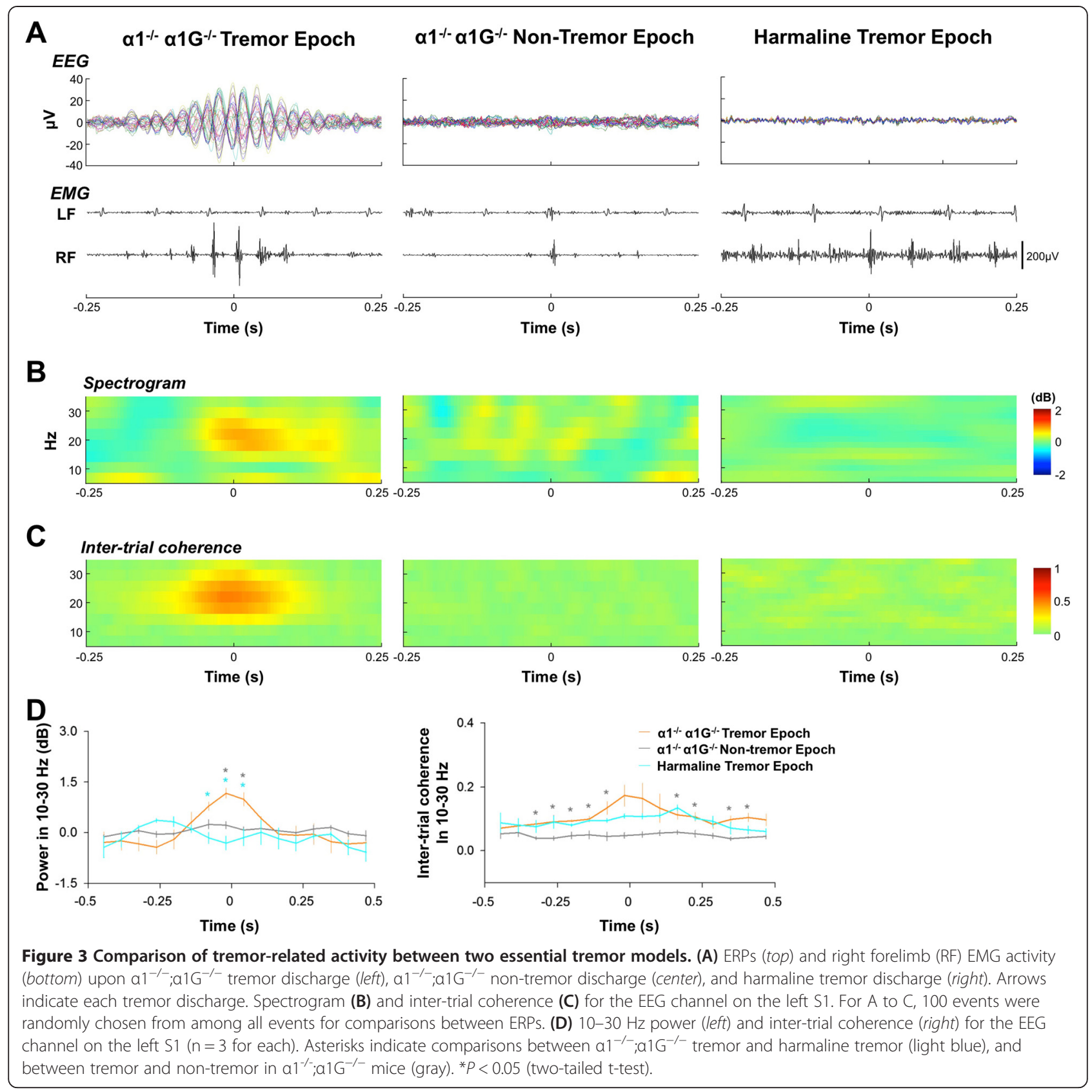

tremor patients [25,26], another clinical study failed to find such a correlation [27], necessitating in-depth comparative studies on cortical involvement in the generation of essential tremor [25-28].

Two action-induced tremor models used in present study has been proposed as an animal models of essential tremor $[4,9]$. By employing event-related potential analysis, we identified TRCPs in $\alpha 1^{-/-} ; \alpha 1 G^{-/-}$mice. However, we failed to find out tremor-related activity in harmaline tremor, suggesting that two models of tremor have different cortical dependency.
Whereas harmaline and $\alpha 1^{-/-}$tremors are ameliorated by propranolol $[29,30], \alpha 1^{-/-} ; \alpha 1 \mathrm{G}^{-/-}$tremor is resistant to propranolol [9]. Deuschl et al. reported that the patients with propranolol susceptibility show synchronous activity between agonist and antagonist muscles during tremor, whereas the patients with propranolol resistance exhibit reciprocal antagonist muscle activity [20]. Notably, harmaline tremor, which is sensitive to propranolol [30], also shows co-contraction of agonist and antagonist muscle pairs during tremor [31]. Thus, it is plausible that the TRCP serves a role in generating propranolol-resistant 
or reciprocal agonist-antagonist contracting subtypes of essential tremor. A comparative study on the cortical activity of essential tremor patients with different phenotypes would effectively test this hypothesis.

The two essential tremor models in this study are also different with respect to their underlying neural circuit mechanism. The Cav3.1 channel is expressed in various motor pathways and may serve different functions [32]. In the thalamocortical pathway, where both Gabral and $C a_{V} 3.1$ genes are co-expressed [33], Cav3.1 may play an inhibitory role in tremorogenesis. In contrast, $\mathrm{Ca}_{\vee} 3.1$ is involved in the generation of tremor in the inferior olive nucleus $[8,32]$. Inferior olive neurons are connected to muscles through the cerebelloreticulospinal pathway $[6,34]$. This means that harmaline could induce tremor while bypassing the involvement of the thalamocortical pathway. Indeed, evidence shows that neither the thalamus nor the motor cortex is associated with harmalineinduced tremor $[7,12]$.

The TRCP in $\alpha 1^{-1-} ; \alpha 1 \mathrm{G}^{-1-}$ mice is not merely a sensory feedback or a movement artifact for a number of reasons. First, it occurred prior to the onset of tremor, at least in some channels, suggesting the possibility that TRCPs are involved in the mechanism responsible for tremor onset (Figure 2). Moreover, TRCPs were not observed in harmaline tremor, which have an intensity similar to that of $\alpha 1^{-1-} ; \alpha 1 \mathrm{G}^{-1-}$ tremor (Figure 3). Although tremoring muscle is known to provide sensory feedback to the cortex, it is not likely that our method is sensitive enough to detect the low level of associated neural activity. These TRCP findings suggest that the two types of tremor are differentially associated with physiological and neural circuit mechanisms.

In conclusion, we have demonstrated the presence of TRCP only in a certain animal model of essential tremor, suggesting its mechanistic heterogeneity. The TRCP characterized in our study could be a useful readout for the diagnosis of essential tremor type. Notably, in patients showing a high level of TRCP, therapeutic strategies that target thalamocortical pathways, rather than T-type $\mathrm{Ca}^{2+}$ channel blockers, might be effective. Furthermore, the $\alpha 1^{-/-} ; \alpha 1 \mathrm{G}^{-/-}$mouse should be a useful model for revealing how the cerebral cortex can generate tremor.

\section{Methods}

\section{Animals}

Animal care and handling conformed to the institutional guidelines of the Animal Care and Use Committee of the Korea Advanced Institute of Science and Technology (KAIST). Mice heterozygous for $\mathrm{GABA}_{\mathrm{A}}$ receptor $\alpha 1$ deletion $\left(\mathrm{\alpha}^{+/-}\right)$in a C57BL/6 J genetic background were obtained from Dr. Gregg E. Homanics at the University of Pittsburgh. Crossing $\alpha 1^{+/-}$and $\alpha 1 G^{+/-}$mice yielded double-heterozygous animals $\left(\alpha 1^{+/-} ; \alpha 1 \mathrm{G}^{+/-}\right)$, which were mated to produce WT $\left(\alpha 1^{+/+} ; \alpha 1 G^{+/+}\right), \alpha 1$ mutant $\left(\alpha 1^{-/-} ; \alpha 1 \mathrm{G}^{+/+}\right)$, and double-mutant $\left(\alpha 1^{-/-} ; \alpha 1 \mathrm{G}^{-/-}\right)$mice. WT mice were utilized for the harmaline-induced tremor model. All experiments were performed using adult mice ( $>10$ weeks old). Mice were housed under a 12-hour light/dark cycle ( 7 a.m. to 7 p.m.) with free access to water and food.

\section{High-resolution EEG}

EEG electrode arrays were prepared as previously described [13]. For surgical anesthesia, ketamine $(100 \mathrm{mg} /$ $\mathrm{kg})$ mixed with xylazine $(5 \mathrm{mg} / \mathrm{kg})$ was administered intraperitoneally. After surgical anesthesia had developed, the head of the mouse was fixed on a stereotaxic apparatus (David Kopf Instruments, CA, USA), and an incision of $\sim 2.5 \mathrm{~cm}$ was made on the scalp. The skull was exposed using micro-clamps, and tissue debris was removed by carefully wiping with a $70 \%$ ethanol-soaked cotton ball. After moisturizing the skull surface with a drop of tap water, a polyamide-based, high-density film electrode was placed on the skull and carefully positioned so that the vertical midline was aligned with the skull midline and the bregma met the upper edge of the third branch. After positioning the electrode, two screws were implanted onto the skull, and dental acrylate was coated over the electrode array. The viscosity of the dental acrylate mix used was carefully examined before application. Mice were allowed to recover under an infrared lamp and housed singly until the experiment.

\section{Electromyography}

The electrodes for electromyography (EMG) in mice were made with Teflon-coated stainless steel wires (A-M Systems, WA, USA) and implanted as previously described [35]. Briefly, two wires were combined and twisted together with a knot $4 \mathrm{~cm}$ from the end. The Teflon insulation was removed from 0.5 -mm-long segments 1 or $2 \mathrm{~mm}$ from the knot in both wires to create two exposures located $1 \mathrm{~mm}$ apart. The ends of both wires were crumpled into the shaft of a 26-gauge needle. The opposite ends of the wires were cut $\sim 8 \mathrm{~cm}$ from the knot, bared, and soldered to a connector. After the fur in the dorsal neck region was shaved away, small incisions were made in the skin above the left and right forelimb muscles (triceps brachii). EMG electrodes were inserted under the skin from the neck incision to the muscles. The needle harboring the electrode was inserted into the target muscle until the knot proximal to the bared regions was firmly butted against the surface of the muscle. The distal end of the electrode exiting the muscle was loosely knotted, and the knot was moved to the muscle surface and tightened. Incisions in the limbs were closed with silk suturing material. 


\section{Analysis of tremor}

After habituation in the recording room for 30 minutes, the mouse was placed on a stainless steel plate $(25 \mathrm{~cm}$ diameter) suspended by two elastic rubber bands. The edge of the plate was surrounded by paper $(5 \mathrm{~cm}$ wide) to prevent mice from falling off. The behavior of the mouse was monitored using a video camera positioned above the platform. For induction of harmaline tremor, harmaline hydrochloride dissolved in $0.9 \%$ saline was injected intraperitoneally (15 mg/kg). A DC response accelerometer (Model No. 3711D1FA3G; Piezotronics, NY, USA) was mounted below the steel plate to aid in tremor detection.

\section{Acquisition of EEG and EMG signals}

High-resolution EEG, EMG, and accelerometer signals were obtained using a SynAmps ${ }^{2}$ amplifier (Neuroscan Inc., TX, USA) with a $2000-\mathrm{Hz}$ sampling rate. Channel 34 of the EEG was used as a reference and channel 33 was used as a ground. Contact impedances of EEG channels were measured using Scan 4.3 software (Neuroscan Inc.) with $30-\mathrm{Hz}$ test pulses. EEG channels with abnormal impedance $(>500 \mathrm{kOhm})$ were excluded from further analyses.

\section{Analysis of tremor-related potentials Pre-processing}

All signal processing and analyses were performed using the Matlab-based open source toolbox EEGLAB [36] or custom Matlab codes. Signals were loaded onto EEGLAB and filtered with a 100-Hz high-pass filter for EMG signals and a 1-200 Hz band-pass filter for EEG and accelerometer signals. All filters used were finite-impulse response filters. EEG signals were re-referenced by common average reference using an EEGLAB plug-in algorithm.

\section{Tremor detection}

EMG traces obtained from the left or right forelimb triceps brachii muscle were used for measuring tremor. Raw EMG traces were rectified and smoothed using a movingaverage window. A cluster of EMG action potentials with peak amplitudes 2.5-fold greater than the standard deviation of baseline EMG activity and separated from others by more than $25 \mathrm{~ms}$ was defined as an 'EMG discharge'. Rhythmic and successive EMG discharges in a specific frequency range $\left(15-30 \mathrm{~Hz}\right.$ for $\alpha 1^{-/-} ; \alpha 1 \mathrm{G}^{-/-}$and $10-15 \mathrm{~Hz}$ for harmaline tremor) were identified as a 'tremor epoch'. Each EMG discharge in a tremor epoch was defined as a 'tremor discharge'. The 'tremor onset' was determined as the onset time of the first tremor discharge in a tremor epoch. Irregular EMG discharges temporally separated from the tremor epoch by more than 1 second in $\alpha 1^{-/-} ; \alpha 1 \mathrm{G}^{-/-}$mice were identified as 'non-tremor discharges'. Tremor onset is used for ERP analysis in
Figure 2. However, we used tremor discharge itself for comparing TRCP in two tremor models (Figure 3) because single tremor epoch is much longer in harmaline tremor so that it was hard to collect enough number of tremor epochs for ERP analysis. Event detection was performed in the left and right forelimb independently using Matlab codes, and was confirmed by visual inspection.

\section{Event-related potential analysis}

EEG channel activity during each event (500 ms preand post-event) was collected, and a baseline correction was applied to the pre-event period for each event and channel. For the calculation of ERPs, the waveforms of collected events ( $>100$ for tremor discharge and nontremor discharge, and $>50$ for tremor onset) were averaged, and the ERP from each channel was plotted in a different color. ERPs of tremor onset, tremor discharge and non-tremor discharge for $\alpha 1^{-/-} ; \alpha 1 G^{-/-}$and harmaline tremor models were calculated, and ERPs of tremor onset and tremor discharge were identified as TRCPs. Tremor events that occurred in only one forelimb at a given moment $(>0.5$ seconds apart from contralateral limb tremor events) were used for ERP calculations. Intertrial coherence is a frequency-domain measure of activity synchronization between event-related trials [36]. Intertrial coherence value indicates degree of consistency of oscillatory phase across trials at certain latency and frequencym [37] and calculated by EEGLab [36].

\section{Statistical analysis}

All data are presented as mean values \pm SEM, and differences between experimental groups were analyzed for statistical significance using Sigmastat 3.1.

\section{Abbreviations \\ $a 1^{-/-}$: mice null for Gabra1; $a^{-/-} ; a 1 G^{-/-}$: mice double-null for Gabra1 and Cacna1g; EEG: Electroencephalography; EMG: Electromyography; ERP: Event-related potential; TRCP: Tremor-related cortical potential.}

\section{Competing interests}

The authors declare that they have no competing interests.

\section{Authors' contributions}

YGP and DSK designed experiments, performed data analysis, and were primarily responsible for preparing the figures and manuscript. JHC, YK, and SHP designed electrophysiological experiment and participated in data analysis. CL and SK performed EEG data analysis. KYC performed pharmacogenetic experiments. All authors read and approved the final manuscript.

\section{Acknowledgements}

This work was supported by grants from the National Leading Research Laboratory Program and KAIST Future Systems Healthcare Project of the Ministry of Science, ICT and Future Planning (No. 20120008795, 2012 K001117, to D. Kim), the Global Frontier R\&D Program (NRF-M1AXA003-2011-0031525, to $\mathrm{J}-\mathrm{H}$ Choi), and Korea Healthcare Technology R\&D Project, Ministry of Health \& Welfare and the Korea Institute of Planning \& Evaluation for Technology in Food, Agriculture, Forestry, and Fisheries (No. HI12C02050101 and 311011-05-3SB020, to S. H. Paek) 


\section{Author details}

${ }^{1}$ Department of Biological Sciences, Korea Advanced Institute of Science and Technology (KAIST), 335 Gwahak-ro, Yuseong-gu, Daejeon 305-701, Republic of Korea. ${ }^{2}$ Center for Neural Science, Division of Life Science, Korea Institute of Science and Technology, Seoul 136-79, Republic of Korea. ${ }^{3}$ Department of Physics, KAIST, Daejeon 305-701, Republic of Korea. ${ }^{4}$ Department of Medical Science and Engineering, KAIST, Daejeon 305-701, Republic of Korea. ${ }^{5}$ Department of Neurosurgery, Hypoxia/Ischemia Disease Institute, Seoul National University College of Medicine, Seoul 110-744, Republic of Korea.

Received: 9 November 2014 Accepted: 6 January 2015

Published online: 15 January 2015

\section{References}

1. Deuschl G, Raethjen J, Lindemann M, Krack P. The pathophysiology of tremor. Muscle Nerve. 2001;24:716-35.

2. Elble RJ. Central mechanisms of tremor. J Clin Neurophysiol. 1996;13:133-44.

3. Deuschl G, Elble RJ. The pathophysiology of essential tremor. Neurology. 1999:54:S14-20.

4. Le Doux M. Harmaline Tremor. Movement Disorders: Genetics and Models 2005;361-68.

5. Poirier L, Sourkes T, Bouvier G, Boucher R, Carabin S. Striatal amines, experimental tremor and the effect of harmaline in the monkey. Brain. 1966;89:37-52.

6. De Montigny C, Lamarre Y. Rhythmic activity induced by harmaline in the olivo-cerebello-bulbar system of the cat. Brain Res. 1973:53:81-95.

7. Lamarre Y, Joffroy A, Dumont M, De Montigny C, Grou F, Lund J. Central mechanisms of tremor in some feline and primate models. Can J Neurol Sci. 1975;2:227-33

8. Park Y-G, Park H-Y, Lee CJ, Choi S, Jo S, Choi H, et al. CaV3. 1 is a tremor rhythm pacemaker in the inferior olive. Proc Natl Acad Sci. 2010;107:10731-6.

9. Chang K-Y, Park Y-G, Park H-Y, Homanics GE, Kim J, Kim D. Lack of CaV3.1 channels causes severe motor coordination defects and an age-dependent cerebellar atrophy in a genetic model of essential tremor. Biochem Biophys Res Commun. 2011:410:19-23.

10. Huguenard J. Low-threshold calcium currents in central nervous system neurons. Annu Rev Physiol. 1996;58:329-48.

11. Kim D, Song I, Keum S, Lee T, Jeong MJ, Kim SS, et al. Lack of the burst firing of thalamocortical relay neurons and resistance to absence seizures in mice lacking a1G T-type Ca2+ channels. Neuron. 2001;31:35-45.

12. Battista AF, Nakatani S, Goldstein M, Anagnoste B. Effect of harmaline in monkeys with central nervous system lesions. Exp Neurol. 1970;28:513-24.

13. Choi JH, Koch KP, Poppendieck W, Lee M, Shin H-S. High resolution electroencephalography in freely moving mice. J Neurophysiol. 2010;104:1825-34.

14. Handy TC. Event-related potentials: a methods handbook. Cambridge, MA: MIT press; 2005.

15. Johnson R. Event-related potential insights into the neurobiology of memory systems. Handbook Neuropsychol. 1995;10:135-135.

16. Luck SJ. An introduction to the event-related potential technique. MA: MIT press Cambridge; 2005.

17. Walter W, Cooper R, Aldridge V, McCallum W, Winter A. Contingent negative variation: an electric sign of sensori-motor association and expectancy in the human brain. Nature. 1964;203:380-4.

18. Lee M, Kim D, Shin H-S, Sung H-G, Choi JH. High-density EEG recordings of the freely moving mice using polyimide-based microelectrode. J Visualized Exp. 2011;47:1-4

19. Louis ED. Essential tremor. Lancet Neurol. 2005:4:100-10.

20. Deuschl G, Lücking C, Schenck E. Essential tremor: electrophysiological and pharmacological evidence for a subdivision. J Neurol Neurosurg Psychiatry. 1987:50:1435-41

21. Jankovic J. Essential tremor: a heterogenous disorder. Mov Disord. 2002;17:638-44.

22. Louis E, Vonsattel J, Honig L, Ross G, Lyons K, Pahwa R. Neuropathologic findings in essential tremor. Neurology. 2006:66:1756-9.

23. Louis ED, Ford B, Barnes LF. Clinical subtypes of essential tremor. Arch Neurol. 2000;57:1194.

24. Testa CM. Key issues in essential tremor genetics research: Where are we now and how can we move forward? Tremor Other Hyperkinetic Movements. 2013;3:1-19.
25. Hellwig B, Häußler S, Schelter B, Lauk M, Guschlbauer B, Timmer J, et al. Tremor-correlated cortical activity in essential tremor. Lancet. 2001;357:519-23.

26. Raethjen J, Govindan R, Kopper F, Muthuraman M, Deuschl G. Cortical involvement in the generation of essential tremor. J Neurophysiol. 2007;97:3219-28.

27. Halliday D, Conway B, Farmer S, Shahani U, Russell A, Rosenberg J. Coherence between low-frequency activation of the motor cortex and tremor in patients with essential tremor. Lancet. 2000;355:1149-53.

28. Schnitzler A, Münks C, Butz M, Timmermann L, Gross J. Synchronized brain network associated with essential tremor as revealed by magnetoencephalography. Mov Disord. 2009;24:1629-35.

29. Kralic JE, Criswell HE, Osterman JL, O'Buckley TK, Wilkie ME, Matthews DB, et al. Genetic essential tremor in $\gamma$-aminobutyric acidA receptor a1 subunit knockout mice. J Clin Investig. 2005;115:774-9.

30. Paterson NE, Malekiani SA, Foreman MM, Olivier B, Hanania T. Pharmacological characterization of harmaline-induced tremor activity in mice. Eur J Pharmacol. 2009;616:73-80.

31. Milner TE, Cadoret G, Lessard L, Smith AM. EMG analysis of harmaline-induced tremor in normal and three strains of mutant mice with Purkinje cell degeneration and the role of the inferior olive. J Neurophysiol. 1995;73:2568-77.

32. Park Y-G, Kim J, Kim D. The potential roles of T-type Ca2+ channels in motor coordination. Front Neural Circuits. 2013;7:172. 1-11.

33. Lein ES, Hawrylycz MJ, Ao N, Ayres M, Bensinger A, Bernard A, et al. Genome-wide atlas of gene expression in the adult mouse brain. Nature. 2006:445:168-76.

34. Lamarre Y, Mercier L-A. Neurophysiological studies of harmaline-induced tremor in the cat. Can J Physiol Pharmacol. 1971;49:1049-58.

35. Pearson K, Acharya H, Fouad K. A new electrode configuration for recording electromyographic activity in behaving mice. J Neurosci Methods. 2005;148:36-42.

36. Delorme A, Makeig S. EEGLAB: an open source toolbox for analysis of single-trial EEG dynamics including independent component analysis. J Neurosci Methods. 2004;134:9-21.

37. Shin Y-W, Krishnan G, Hetrick WP, Brenner CA, Shekhar A, Malloy FW, et al. Increased temporal variability of auditory event-related potentials in schizophrenia and Schizotypal Personality Disorder. Schizophr Res. 2010;124:110-8.

\section{Submit your next manuscript to BioMed Central and take full advantage of:}

- Convenient online submission

- Thorough peer review

- No space constraints or color figure charges

- Immediate publication on acceptance

- Inclusion in PubMed, CAS, Scopus and Google Scholar

- Research which is freely available for redistribution 